\title{
Arterial thromboembolism in patients with sick sinus syndrome: prediction from pacing mode, atrial fibrillation, and echocardiographic findings
}

\author{
H R Andersen, J C Nielsen, P E B Thomsen, L Thuesen, A K Pedersen, P T Mortensen, \\ $\mathrm{T}$ Vesterlund
}

\begin{abstract}
Objective-To evaluate whether thromboembolism in sick sinus syndrome can be predicted by pacing mode, atrial fibrillation, or echocardiographic findings.

Methods-Patients were randomised to single chamber atrial $(n=110)$ or ventricular $(n=115)$ pacing. They were divided into subgroups with and without brady-tachy syndrome at time of randomisation. The occurrence of atrial fibrillation and thromboembolism during follow up were investigated and compared with echocardiographic findings.

Results-The annual risk of thromboembolism was $5.8 \%$ in patients with bradytachy syndrome randomised to ventricular pacing, $3.2 \%$ in patients without brady-tachy syndrome randomised to ventricular pacing, $3 \%$ in patients with brady-tachy syndrome randomised to atrial pacing, and $1.5 \%$ in patients without brady-tachy syndrome randomised to atrial pacing. In atrial paced patients without brady-tachy syndrome at randomisation and without atrial fibrillation during follow up, the annual risk of thromboembolism was $1.4 \%$. Left atrial size measured by $M$ mode echocardiography was of no value in predicting thromboembolism.

Conclusions-Arterial thromboembolism in patients with sick sinus syndrome is very common and is associated primarily with brady-tachy syndrome at randomisation and with ventricular pacing. The risk of thromboembolism is small in atrial paced patients in whom atrial fibrillation has never been documented. (Heart 1999;81:412-418)
\end{abstract}

Department of Cardiology, Skejby

Sygehus, Aarhus University Hospital, 8200 Aarhus N,

Denmark

H R Andersen

J C Nielsen

P E B Thomsen

L Thuesen

A K Pedersen

P T Mortensen

$\mathrm{T}$ Vesterlund

Correspondence to:

Dr Andersen.

Accepted for publication 25 November 1998
Keywords: sick sinus syndrome; atrial pacing; atrial fibrillation; thromboembolism

In a prospective randomised trial comparing single chamber atrial and ventricular pacing in patients with sick sinus syndrome, we recently found that during long term follow up atrial pacing was associated with significantly less atrial fibrillation, less thromboembolism, and less dilatation of the left atrium compared with ventricular pacing. ${ }^{12}$ Thus theoretically it should be possible to identify groups of patients with either a high risk or a low risk of thromboembolism using ECG and echocardiography. In this way it might be possible to iden- tify subgroups of patients who would benefit from anticoagulation, or who could be left without anticoagulation. The aim of the present post hoc analysis was to study in detail the association between these variables to evaluate whether thromboembolic events could be predicted by pacing mode, atrial fibrillation, or echocardiographic findings.

\section{Methods}

PROTOCOL

The study design has been published in detail previously. ${ }^{13}$ The trial was conducted as a one centre study at Skejby University Hospital. All patients who were referred for treatment with their first pacemaker during the recruitment period from 15 May 1988 to 31 December 1991 were evaluated for randomisation. The patients were asked to participate in the trial if the inclusion criteria (symptomatic bradycardia of less than 50 beats/min or symptomatic QRS pauses of more than 2 seconds) and none of the exclusion criteria were met. ${ }^{3}$ Brady-tachy syndrome at inclusion in the study was defined as bradycardia and at least one documented episode of a supraventricular tachyarrhythmia. ${ }^{4}$ Patients with brady-tachy syndrome were included in the study if atrial tachycardia was present for $\leqslant 50 \%$ of the time before implantation. ${ }^{3}$ The time before implantation was "defined" as the preimplant period (days, weeks, months) where ECG or Holter documentation had been obtained. This period was usually only a few days and seldom longer than one week. After giving informed consent, patients were randomised to treatment with single chamber atrial or ventricular pacing.

Medical history, physical examination, and echocardiography were carried out before implantation. Follow up visits were after three months, 12 months, and subsequently once a year. The follow up visit included physical examination, 12 lead standard ECG recording, pacemaker check, and measurement of left atrial diameter and left ventricular dimensions by $\mathrm{M}$ mode echocardiography. Follow up evaluation was not blinded.

In 1995 it was decided that the last patient included should be followed for at least five years before final data analysis, which was determined to be 31 December 1996.

ATRIAL FIBRILLATION

Atrial fibrillation was diagnosed by 12 lead standard ECG at the follow up visits (not from ECG records taken at any other time). Atrial 
fibrillation was categorised as chronic if it was recorded at two consecutive follow up visits and no sinus rhythm was observed subsequently. In case of atrial fibrillation, conversion to normal rhythm was always considered, and a decision to attempt medical conversion or dc conversion was taken from clinical criteria.

THROMBOEMBOLISM

Stroke and peripheral arterial embolus were classified as thromboembolic events. Stroke was diagnosed when neurological symptoms of presumably cerebral ischaemic origin persisted for more than 24 hours or if patients died within 24 hours from an acute cerebrovascular event. The decision to verify the diagnosis of stroke by computed tomography of the brain was made on clinical grounds when the patient had been admitted to hospital with the cerebral event. Peripheral arterial embolus was diagnosed if verified at embolectomy or necropsy. Information about thromboembolic events was obtained by medical history and physical examination at the follow up visits. If an event had occurred, additional information was obtained from the patient's doctor and from hospital files to validate the diagnosis. Thromboembolic events were classified as first events or re-events and as fatal or non-fatal.

\section{ECHOCARDIOGRAPHY}

Left atrial diameter and left ventricular end systolic (LVESD) and end diastolic diameters (LVEDD) were determined by $\mathrm{M}$ mode echocardiography in accordance with the recommendations of the American Society of Echocardiography. ${ }^{5}$ Left ventricular dimensions were obtained at the level of the chordae. Measurements were done by the leading edge method. Left ventricular fractional shortening was calculated as (LVEDD - LVESD)/ LVEDD. Left atrial diameter at inclusion in the study and at last follow up were compared between patients with and without thromboembolic events during the follow up period. The number of patients with a left atrial diameter of more than $40 \mathrm{~mm}$ measured once or more during follow up was recorded and compared between groups with and without thromboembolic events. Left ventricular end diastolic diameter and fractional shortening at recruitment were compared between subgroups with and without thromboembolic events.

\section{ANTICOAGULATION AND ASPIRIN}

The decision to start treatment with anticoagulation or aspirin during the follow up period was taken from clinical criteria. Generally, paroxysmal atrial fibrillation was not an indication for anticoagulation. If atrial fibrillation did not convert spontaneously or after the administration of drugs or dc shock, the patient's general practitioner was advised to start anticoagulation, if there was no contraindication to such treatment. The international normalised ratio (INR) target range was 2.0-3.0 during anticoagulation. The dose of aspirin used during the study was $100-150 \mathrm{mg}$ once a day.
ETHICS

The study was approved by the national Danish ethics committee and was conducted in accordance with the rules of the Helsinki declaration.

STATISTICAL ANALYSIS

All statistical analyses comparing the atrial and ventricular groups were done according to the intention to treat principle. Continuous variables are expressed as mean (SD). Treatment groups were compared by $\chi^{2}$ test (two tailed) for discrete variables and by two tailed Student's $t$ test for continuous variables. In patients with and without brady-tachy syndrome, Kaplan-Meier plots were calculated for freedom from atrial fibrillation, chronic atrial fibrillation, and arterial thromboembolism, and compared by log-rank test. Kaplan-Meier plots were calculated for freedom from arterial thromboembolism in patients with brady-tachy syndrome or/and atrial fibrillation during follow up and in patients without atrial fibrillation before implantation and during follow up, and compared by log-rank test. Relative risk and $95 \%$ confidence intervals (CI) were calculated by the univariate Cox proportional hazards method. A p value less than 0.05 was considered significant. SPSS for Windows was used for the statistical analysis.

\section{Results}

\section{PATIENTS}

In all, 225 patients (142 women, 83 men, age 76 (8) years, range 50 to 92 years) were randomised to atrial pacing $(n=110)$ and ventricular pacing $(\mathrm{n}=115)$, respectively. Brady-tachy syndrome was present in 43 of the patients in the atrial group and in 51 of the patients in the ventricular group. Baseline characteristics in the two groups, divided into groups with and without brady-tachy syndrome, are presented in table 1. No patients were lost to follow up. Mean follow up was 5.5 (2.4) years for the total study population, and was equal in the atrial group (5.7 (2.3) years) and the ventricular group (5.3 (2.5) years).

Ninety four of the 110 patients randomised to atrial pacing $(85 \%)$ were treated as randomised during follow up. Of these 110 patients, six patients had ventricular leads at primary implantation. During follow up, another five patients had ventricular leads, and in five patients the pacemaker was upgraded to a dual chamber system (in four cases because of atrioventricular block). In the ventricular group, 109 patients (95\%) were treated as randomised during follow up. All patients randomised to ventricular pacing were discharged from hospital with ventricular pacing. During follow up, one patient had the pacing system changed to atrial pacing, in another three patients, upgrading to a dual chamber system was necessary, and two patients had the pacemaker system explanted. ${ }^{1}$

Medical treatment was similar during follow up in the two groups, except for the dose of diuretics, which increased more in the ventricular group than in the atrial group. ${ }^{2}$ 
Table 1 Baseline characteristics

\begin{tabular}{|c|c|c|c|c|}
\hline & \multicolumn{2}{|l|}{ Atrial group } & \multicolumn{2}{|c|}{ Ventricular group } \\
\hline & BTS- & BTS+ & BTS- & $B T S+$ \\
\hline Number of patients & 67 & 43 & 64 & 51 \\
\hline Age (years) & $76(9)$ & $76(8)$ & $76(9)$ & $75(8)$ \\
\hline $\operatorname{Sex}(F / M)$ & $41 / 26$ & $32 / 11$ & $37 / 27$ & $32 / 19$ \\
\hline \multicolumn{5}{|l|}{ Blood pressure $(\mathrm{mm} \mathrm{Hg})$} \\
\hline Systolic & $145(20)$ & $146(26)$ & $145(22)$ & $143(29)$ \\
\hline Diastolic & $76(10)$ & $79(11)$ & $78(11)$ & $79(12)$ \\
\hline \multicolumn{5}{|l|}{ Symptoms indicating need for pacemaker } \\
\hline Syncope & 31 & 23 & 32 & 32 \\
\hline Dizzy spells & 33 & 18 & 30 & 19 \\
\hline Heart failure & 3 & 2 & 2 & 0 \\
\hline \multicolumn{5}{|l|}{ Arrhythmia indicating need for pacemaker } \\
\hline Sinus bradycardia & 18 & & 18 & \\
\hline Sinoatrial block & 49 & & 46 & \\
\hline \multicolumn{5}{|l|}{ Clinical events before randomisation } \\
\hline Angina pectoris & 15 & 14 & 19 & 11 \\
\hline Myocardial infaction & 6 & 6 & 9 & 9 \\
\hline Stroke & 2 & 5 & 4 & 3 \\
\hline Arterial embolus & 2 & 0 & 0 & 2 \\
\hline Transient cerebral ischaemia & 6 & 2 & 0 & 2 \\
\hline Heart valve disease & 2 & 1 & 1 & 1 \\
\hline NYHA class I/II/III/IV & $51 / 12 / 4 / 0$ & $28 / 12 / 3 / 0$ & $50 / 12 / 2 / 0$ & $42 / 8 / 1 / 0$ \\
\hline \multicolumn{5}{|l|}{$M$ mode echocardiography ${ }^{\star}$} \\
\hline Left atrial diameter (mm) & $34(7)$ & $34(5)$ & $34(6)$ & $34(7)$ \\
\hline Left ventricular fractional shortening & $0.36(0.11)$ & $0.33(0.16)$ & $0.37(0.12)$ & $0.35(0.12)$ \\
\hline \multicolumn{5}{|l|}{ Medical treatment } \\
\hline Digoxin & 5 & 17 & 2 & 9 \\
\hline$\beta$ Blocker & 3 & 4 & 1 & 0 \\
\hline Calcium blocker & 3 & 10 & 7 & 4 \\
\hline Antiarrhythmic drugs & 5 & 7 & 3 & 3 \\
\hline Frusemide (mg/day) & $23(44)$ & $46(60)$ & $18(29)$ & $30(47)$ \\
\hline Aspirin & 26 & 22 & 19 & 27 \\
\hline Warfarin & 4 & 2 & 0 & 1 \\
\hline
\end{tabular}

Values are numbers of patients. Continuous data are presented as means (SD).

${ }^{\star}$ Measurements represent data from 221 patients because of an inadequate echocardiographic window in four patients.

BTS, brady-tachy syndrome; NYHA, New York Heart Association heart failure classification.

ATRIAL FIBRILLATION

Atrial fibrillation and chronic atrial fibrillation occurred less often in the atrial group than in the ventricular group during follow up (relative risk $0.54,95 \%$ CI 0.33 to $0.89, \mathrm{p}=0.012$ for atrial fibrillation; $0.35,95 \%$ CI 0.16 to 0.76 , $\mathrm{p}=0.004$, for chronic atrial fibrillation). In both the ventricular and the atrial groups, brady-tachy syndrome at randomisation was associated with an increased risk of atrial fibrillation (relative risk $2.14,95 \%$ CI 1.14 to 4.01 , $\mathrm{p}=0.015$ in the ventricular group; $2.76,95 \%$ CI 1.26 to $6.05, \mathrm{p}=0.008$, in the atrial group), and an increased risk of chronic atrial fibrilla-

Table 2 Baseline variables, left atrial diameter during follow up, and thromboembolism during follow up

\begin{tabular}{lll}
\hline & Thromboembolism & No thromboembolism \\
\hline Ventricular group & & \\
Number & 26 & 89 \\
Diastolic blood pressure (mm Hg) & $76(11)$ & $79(11)$ \\
Systolic blood pressure (mm Hg) & $143(27)$ & $144(25)$ \\
LVEDD on entry (mm) & $48(8)$ & $49(7)$ \\
LV fractional shortening on entry & $0.34(0.12)$ & $0.37(0.12)$ \\
Left atrial diameter on entry (mm) & $34(6)$ & $34(7)$ \\
Left atrial diameter at last FU (mm) & $40(7)$ & $41(7)$ \\
Left atrial diameter > 40 mm during FU (n) & 19 & 61 \\
NYHA class I/II/III/IV & $23 / 3 / 0 / 0$ & $69 / 17 / 3 / 0$ \\
Atrial group & & \\
Number & 13 & 97 \\
Diastolic blood pressure (mm Hg) & $82(12)$ & $77(10)$ \\
Systolic blood pressure (mm Hg) & $155(27)$ & $145(21)$ \\
LVEDD on entry (mm) & $49(5)$ & $47(8)$ \\
LV fractional shortening on entry & $0.32(0.08)$ & $0.36(0.14)$ \\
Left atrial diameter on entry (mm) & $37(6)$ & $34(6)$ \\
Left atrial diameter at last FU (mm) & $37(8)$ & $37(7)$ \\
Left atrial diameter > 40 mm during FU (n) & 10 & 51 \\
NYHA class I/II/III/IV & $7 / 5 / 1 / 0$ & $72 / 19 / 6 / 0$ \\
\hline
\end{tabular}

Values are mean (SD) unless otherwise stated. There were no significant differences between patients with and without thromboembolism.

FU, follow up; LV, left ventricular; LVEDD, left ventricular end diastolic diameter; NYHA, New York Heart Association functional classification. tion (relative risk $2.83,95 \%$ CI 1.18 to 6.79 , $\mathrm{p}=0.015$ in the ventricular group; $3.79,95 \%$ CI 0.94 to $15.3, \mathrm{p}=0.05$, in the atrial group). In patients without brady-tachy syndrome at randomisation, the occurrence of atrial fibrillation-and of chronic atrial fibrillationwas less likely in the atrial group than in the ventricular group (relative risk 0.67, 95\% CI 0.46 to $0.98, p=0.03$ for atrial fibrillation; $0.52,95 \%$ CI 0.27 to $1.01, p=0.03$, for chronic atrial fibrillation), whereas there was no significant difference in patients with bradytachy syndrome (relative risk for the atrial group $0.83,95 \%$ CI 0.60 to $1.15, p=0.24$ for atrial fibrillation; and $0.67,95 \%$ CI 0.42 to $1.09, \mathrm{p}=0.09$ for chronic atrial fibrillation).

\section{ARTERIAL THROMBOEMBOLISM}

There were no significant differences in baseline variables between patients with and without thromboembolism during follow up (table 2). Overall, arterial thromboembolism occurred in 13 patients in the atrial group and in 26 patients in the ventricular group (relative risk for atrial group $0.47,95 \%$ CI 0.24 to 0.92 , $\mathrm{p}=0.023$ ). Considering patients with and without brady-tachy syndrome separately, there were no significant differences in occurrence of thromboembolism between the atrial and ventricular groups (fig 1). The risk of thromboembolism in the various subgroups is presented in table 3 .

Of the 13 patients with thromboembolism in the atrial group, 11 had one stroke each, one had a peripheral embolus, and one had two strokes. Of the 26 patients with thromboembolism in the ventricular group, 14 had one stroke 


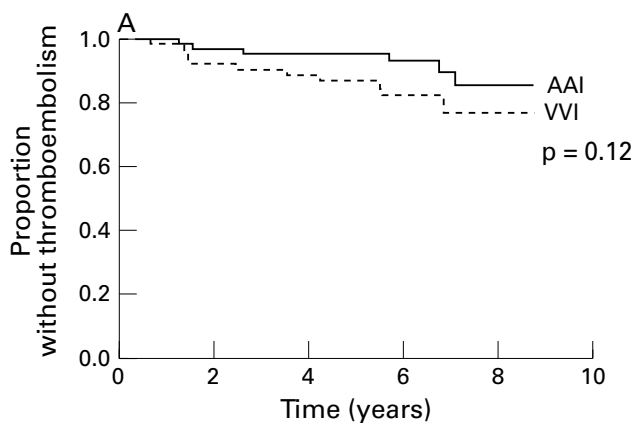

AAl: $\quad \begin{array}{lllllllll}67 & 63 & 58 & 54 & 52 & 50 & 37 & 23 & 10\end{array}$

VVI: $\quad \begin{array}{lllllllll}64 & 60 & 54 & 52 & 48 & 45 & 29 & 12 & 4\end{array}$

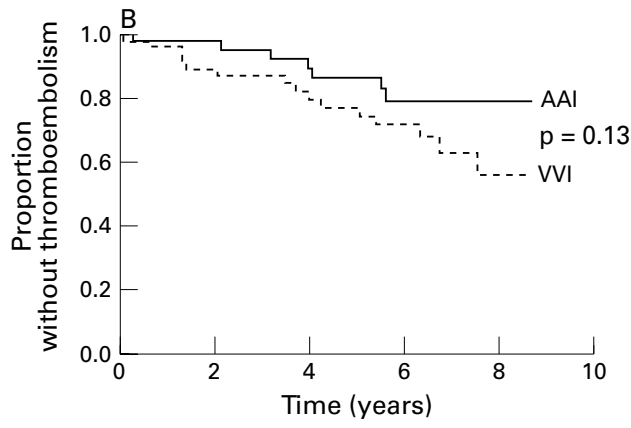

AAl: $\quad \begin{array}{llllllllll}43 & 39 & 38 & 35 & 31 & 29 & 19 & 11 & 2\end{array}$

VVI: $\quad \begin{array}{llllllllll}51 & 42 & 39 & 35 & 32 & 30 & 21 & 12 & 7\end{array}$

Figure 1 Kaplan-Meier plots of freedom from arterial thromboembolism during follow up in patients randomised to single chamber atrial ( $A A I)$ or ventricular (VVI) pacing stratified in groups without brady-tachy syndrome $(A)$ and with brady-tachy syndrome $(B)$ at randomisation. Figures below abscissa indicate number of patients at risk during follow up.

each, five had one peripheral embolus each, five had two strokes each, one had two strokes and one peripheral embolus, and one had two strokes and two peripheral emboli. Thus a total of 14 thromboembolic events occurred in the atrial group versus 36 events in the ventricular group (fig 2). There were five fatal thromboembolic events in the atrial group (two with brady-tachy syndrome) versus 14 (seven with brady-tachy syndrome) in the ventricular group (relative risk $0.34,95 \%$ CI 0.12 to 0.96 , $\mathrm{p}=0.032)$.

In a total of 41 strokes, the diagnosis was based on clinical findings in 29 cases while nine strokes were verified by computed tomography and three at necropsy. Eight peripheral emboli were verified by embolectomy (four from the arms and four from the legs), and one embolus was verified at necropsy (located in an abdominal artery).

Table 3 Risk of thromboembolism during follow up

\begin{tabular}{lllll}
\hline Randomisation & $\begin{array}{l}\text { BTS at } \\
\text { randomisation }\end{array}$ & $\begin{array}{l}\text { Patients with } \\
\text { event/all patients }\end{array}$ & $\begin{array}{l}\text { Years observed } \\
\text { (mean (SD)) }\end{array}$ & $\begin{array}{l}\text { Risk } \\
\text { (\% per year) }\end{array}$ \\
\hline Ventricular pacing & Yes & $15 / 51$ & $5.1(2.7)$ & 5.8 \\
Ventricular pacing & No & $11 / 64$ & $5.4(2.2)$ & 3.2 \\
Atrial pacing & Yes & $7 / 43$ & $5.4(2.4)$ & 3.0 \\
Atrial pacing & No & $6 / 67$ & $5.8(2.3)$ & 1.5 \\
\hline
\end{tabular}

BTS, brady-tachy syndrome.
THROMBOEMBOLISM AND ATRIAL FIBRILLATION The patients were stratified into two groups: one without brady-tachy syndrome at randomisation and without atrial fibrillation during follow up (patients in whom atrial fibrillation was never documented) $(\mathrm{n}=102,56$ single chamber atrial pacing (AAI)); and one group with brady-tachy syndrome at randomisation and/or atrial fibrillation during follow up $(\mathrm{n}=123,54 \mathrm{AAI})$. In patients in whom atrial fibrillation was never documented, thromboembolism was significantly less common in the atrial group than in the ventricular group (relative risk $0.46,95 \%$ CI 0.24 to 0.90 , $\mathrm{p}=0.013$ ) (fig 3). In patients with brady-tachy syndrome and/or atrial fibrillation during follow up, occurrence of thromboembolism was not significantly different between treatment groups (fig 3).

Of the 39 patients who developed arterial thromboembolism during follow up, 12 never had atrial fibrillation, either before randomisation or during follow up. Of the eight patients with more than one thromboembolic event, six had brady-tachy syndrome at randomisation but none had atrial fibrillation during follow up before the first thromboembolic episode, and six never had atrial fibrillation during follow up. Both patients with more than two thromboembolic events had brady-tachy syndrome at randomisation. The patient with three events had developed chronic atrial fibrillation at the one year follow up visit, whereas the patient with four events never had atrial fibrillation during follow up.

In order to describe a group of patients that can be identified in every pacemaker clinic, we analysed separately the patients who were treated as randomised and who had no atrial fibrillation before randomisation or during follow up. Four of 50 patients in the atrial group (risk/year $1.4 \%$ ) and nine of 44 patients in the ventricular group (risk/year 4.2\%) had thromboembolic events, and two re-events occurred in the ventricular group.

LEFT ATRIAL DIAMETER AND THROMBOEMBOLISM There were no significant differences in left atrial diameter between the patients who developed thromboembolism and those who did not (table 2), nor was there any difference in the proportion of patients with a left atrial diameter of more than $40 \mathrm{~mm}$ during follow up between these two groups (table 2). Thus thromboembolic events were not preceded by an excessive increase in left atrial diameter.

\section{ANTICOAGULATION AND ASPIRIN}

During follow up, warfarin was given to 10 patients in the atrial group compared with 16 patients in the ventricular group. Mean age at inclusion in the study was 70 (7) years in the 26 patients treated with warfarin versus 76 (8) years in the remaining 199 patients $(p<0.001)$. In the group of patients with atrial fibrillation during follow up, eight of 26 patients in the atrial group and 13 of 40 in the ventricular group were treated with warfarin. 


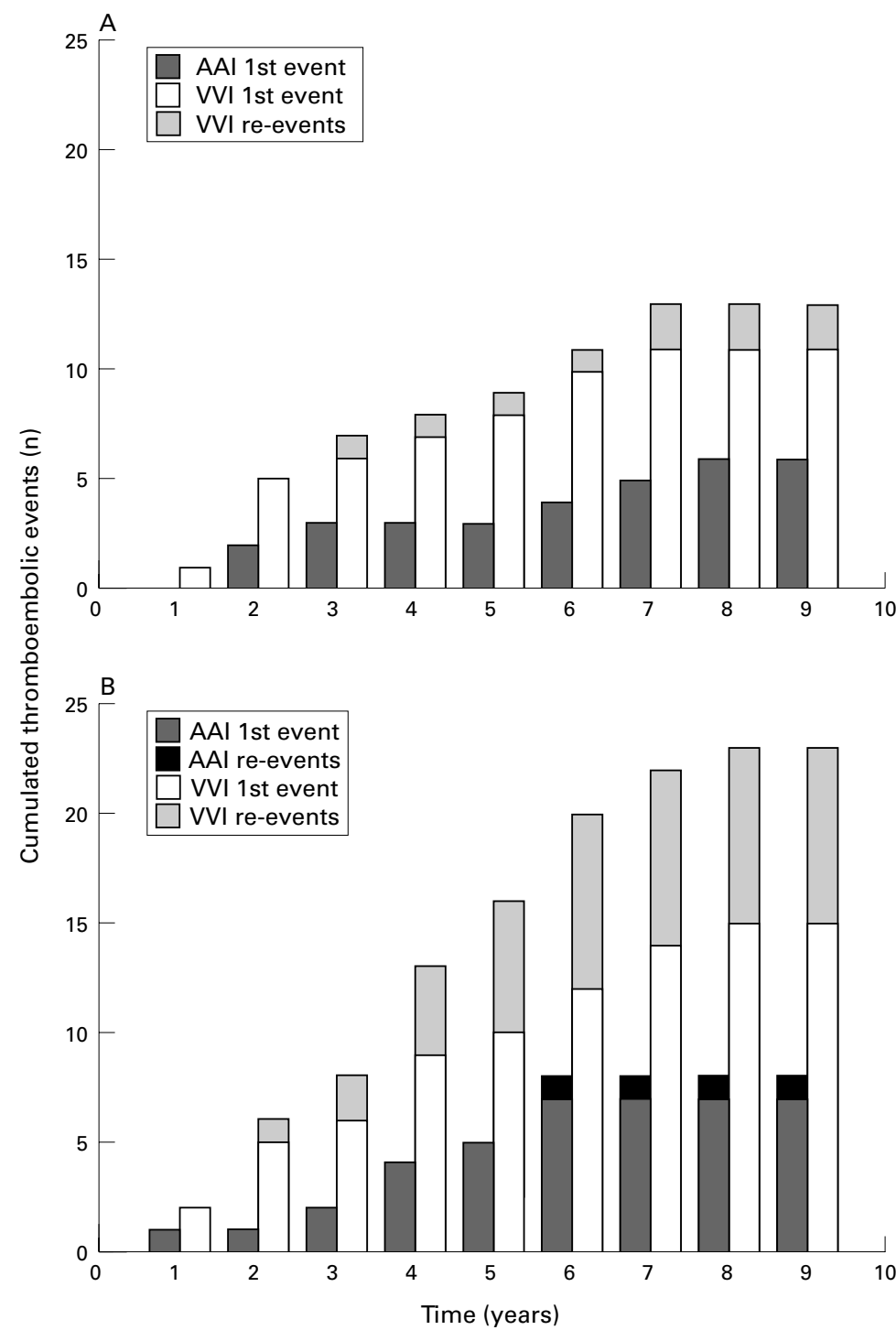

Figure 2 Cumulative number of arterial thromboembolic events during follow up in patients without brady-tachy syndrome at randomisation $(A)$ and in patients with brady-tachy syndrome at randomisation (B) in the two treatment groups. AAI, single chamber atrial pacing; VVI, single chamber ventricular pacing.

Among patients who developed thromboembolism, four of 13 patients in the atrial group and seven of 26 in the ventricular group were treated with warfarin. In only one of the nine patients in whom atrial fibrillation was observed during follow up before the first thromboembolic event was warfarin started before this event. In the eight patients with more than one thromboembolic episode, four were treated with warfarin.

During follow up, aspirin was given to 81 patients in the ventricular group and to 69 patients in the atrial group. Among patients with thromboembolism, 11 patients in the atrial group and 17 in the ventricular group were treated with aspirin. In patients with more than one thromboembolic episode, aspirin treatment was given to seven of eight patients.

\section{Discussion}

Our analysis shows that arterial thromboembolism in patients with sick sinus syndrome occurs very commonly and is associated with

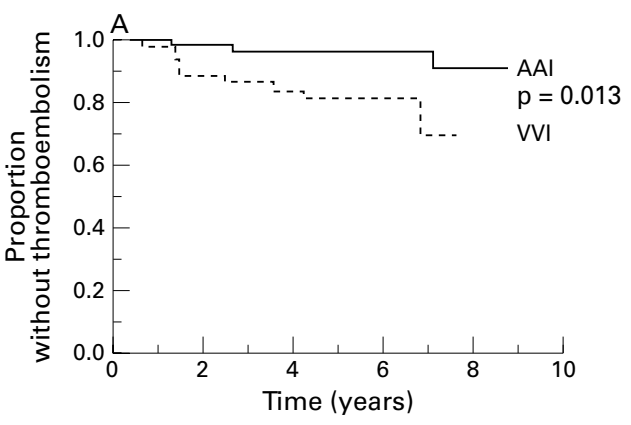

AAl: $\quad \begin{array}{lllllllll}56 & 52 & 49 & 45 & 43 & 41 & 30 & 19 & 9\end{array}$

VVI: $\quad \begin{array}{lllllllll}46 & 42 & 37 & 35 & 31 & 29 & 20 & 4 & 0\end{array}$

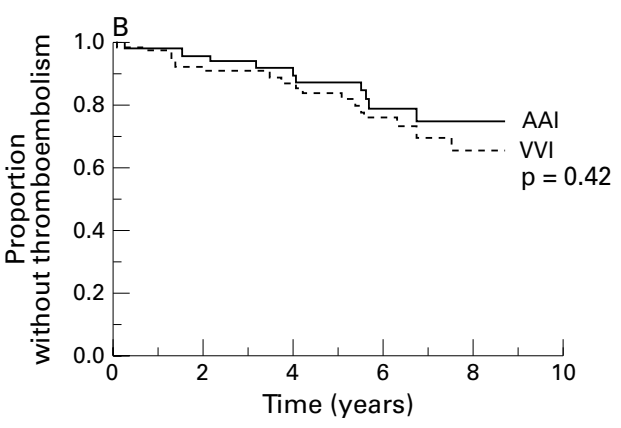

AAl: $\quad \begin{array}{lllllllll}54 & 50 & 47 & 44 & 40 & 38 & 26 & 15 & 3\end{array}$

VVI: $\quad \begin{array}{llllllllll}69 & 60 & 56 & 52 & 49 & 46 & 30 & 20 & 11\end{array}$

Figure 3 Kaplan-Meier plots of freedom from arterial thromboembolism during follow up in patients randomised to single chamber atrial (AAI) or ventricular (VVI) pacing stratified into two groups: patients without brady-tachy syndrome at randomisation and without atrial fibrillation during follow up $(A)$, and patients with brady-tachy syndrome at randomisation and/or atrial fibrillation during follow up (B).

the presence of brady-tachy syndrome at randomisation, with the selection of ventricular pacing mode, and with the occurrence of atrial fibrillation during follow up, whereas $M$ mode echocardiography is of no particular value in predicting thromboembolism in this population. Furthermore, our study shows that a large group of patients can be identified who are at low risk of thromboembolism - that is, patients randomised to and treated with atrial pacing in whom atrial fibrillation was never documented. These patients can probably be left without anticoagulation whereas in all other patients with sick sinus syndrome, anticoagulants should seriously be considered, even though the patients are in sinus rhythm. All patients with sick sinus syndrome treated with a ventricular pacemaker should be considered as candidates for anticoagulation treatment; alternatively, upgrading of the pacemaker to an atrial system should be considered.

ATRIAL FIBRILLATION

In this trial, atrial fibrillation and chronic atrial fibrillation were more common in the ventricular group than in the atrial group, but the differences between groups were significant only in patients without brady-tachy syndrome at randomisation. Thus ventricular pacing apparently provokes atrial fibrillation, or atrial pacing can prevent or delay atrial fibrillation, in patients with sick sinus syndrome, and the 
effect of the pacing mode seems to be strongest in patients without brady-tachy syndrome. These results are in contrast to findings in a large long term observational study of 507 patients with sick sinus syndrome treated with physiological pacemakers (DDD, $\mathrm{n}=376$; AAI, $n=19$ ) or ventricular (VVI) pacemakers $(\mathrm{n}=112) .{ }^{6}$ In that study, none of 132 patients without brady-tachy syndrome at implantation had developed chronic atrial fibrillation after five years of follow up whatever the pacing mode, whereas in patients with brady-tachy syndrome, 86 (23\%) developed chronic atrial fibrillation during a mean follow up of 59 months. Ventricular pacing was an independent predictor of chronic atrial fibrillation in this subgroup. The investigators were apparently able to identify patients at high risk of atrial fibrillation by a more intensive search for atrial fibrillation before implantation. Using extensive Holter and telemetry monitoring, they identified a large proportion of patients $(74 \%)$ with brady-tachy syndrome in their study, which is higher than in other studies $(27 \%$ to $58 \%),{ }^{7-11}$ including our present one $(42 \%)$. They may also have been more aggressive in re-establishing sinus rhythm because of the lower age of their patients (mean age 66 years).

THROMBOEMBOLISM

There is a definite risk of thromboembolism in patients with sick sinus syndrome, both before and after implantation of a pacemaker. ${ }^{12-16}$ In our present trial, the risk of thromboembolism was high $(5.8 \% /$ year $)$ in patients with bradytachy syndrome randomised to ventricular pacing, whereas the risk was only $1.5 \%$ /year in patients without brady-tachy syndrome randomised to atrial pacing. Thus patients with brady-tachy syndrome and ventricular pacing have a very high risk of thromboembolism, similar to patients with chronic atrial fibrillation not treated with anticoagulation. ${ }^{17}$ Moreover, thromboembolic re-events occurred almost exclusively in this group of patients with brady-tachy syndrome treated with ventricular pacing. Furthermore, patients treated with ventricular pacemakers also had a considerable risk of thromboembolism without atrial fibrillation. These findings indicate that patients already treated with ventricular pacemakers could benefit from anticoagulation even though they are in sinus rhythm.

Our findings of a higher incidence of thromboembolism in the ventricular group than in the atrial group confirm reports from most observational studies. ${ }^{6} 1011141819$ However, some of these retrospective studies have reported a much lower overall incidence of thromboembolism, ${ }^{6}{ }^{10}$ probably because some of the thromboembolic events were not recorded in the pacemaker files, which often focus largely on pacemaker indices.

Left atrial dilatation was not predictive of thromboembolism in our trial, in accordance with the findings of Sgarbossa et al in patients with sick sinus syndrome, ${ }^{6}$ but in contrast to findings in non-paced patients with atrial fibrillation $^{20}{ }^{21}$ and in the general population. ${ }^{22}$
However, patients with sick sinus syndrome treated with pacemakers form a selected population in which the factors causing thromboembolism might be different from those in the general population and in patients with atrial fibrillation.

Our findings suggest that ventricular pacing might increase the risk of thromboembolism in sick sinus syndrome by mechanisms other than atrial fibrillation. Such additional mechanisms are not yet fully elucidated, but ventricular pacing has been associated with increased platelet aggregability, ${ }^{23}$ platelet activation, ${ }^{24}$ and left atrial appendage dysfunction, ${ }^{25}{ }^{26}$ all of which could increase the risk of thromboembolism. Preliminary reports have indicated that more aggressive inhibition of platelets by ticlopidine can influence thromboembolism. ${ }^{27}$

The high risk of thromboembolism associated with ventricular pacing indicates that this type of pacing should be abandoned in patients with sick sinus syndrome. Our results also suggest that anticoagulation should be considered in all patients with brady-tachy syndrome despite the mode of pacemaker treatment. The only patients who can probably be left without anticoagulants are those with atrial pacemakers in whom atrial fibrillation has never been observed.

\section{ANTICOAGULATION}

Anticoagulants have proved effective in preventing thromboembolic complications in nonpaced patients with chronic atrial fibrillation, ${ }^{17}$ and in patients with paroxysmal atrial fibrillation. ${ }^{21}{ }^{28-30}$ In our trial, anticoagulation (warfarin) was given only to a minority of patients with atrial fibrillation during follow up. Thus anticoagulants were underused in our population during the study period, considering the current recommendations for such treatment. ${ }^{31}$ During the conduct of our trial, paroxysmal atrial fibrillation was not regarded an indication for anticoagulation. Anticoagulants appear to have been prescribed mainly for the younger of our patients, and since most were elderly and had relative or absolute contraindications for anticoagulation when chronic atrial fibrillation occurred, this restricted the numbers treated. This underutilisation of anticoagulants is in accord with several studies in non-paced populations. ${ }^{32-36} \mathrm{In}$ some of these, the underutilisation of anticoagulants was also most marked in the older patients, ${ }^{32} 34$ even though it is now known that the risk of thromboembolism increases with advancing age. ${ }^{32} 37$

Aspirin was given to two thirds of our patients, and the majority of those who developed thromboembolism in the present trial were treated with aspirin at the time of the thromboembolic event. Thus aspirin treatment (100-150 mg/day) apparently had no effect in these patients, in accordance with findings in non-paced patients with atrial fibrillation. ${ }^{17} 38$

\section{LIMITATIONS}

Post hoc subgroup analyses are at best hypothesis generating, and should be considered with caution. The subgroup analysis of patients with 
and without brady-tachy syndrome was not predefined before start of the study; thus the study might not be powered to detect differences between subgroups. However, the present analysis serves as a basis for future prospective trials - for example testing the effect of upgrading ventricular pacing to atrial pacing in patients with sick sinus syndrome.

Anticoagulants were underused during our study, taking into account the current guidelines on anticoagulation. Anticoagulation might reduce or eliminate the excess risk of thromboembolism in patients with sick sinus syndrome treated by ventricular pacing. However, several studies have shown that in daily clinical practice anticoagulation is often underused, ${ }^{32-36}$ especially in the elderly. ${ }^{32} 34$

\section{CONCLUSION}

Arterial thromboembolism in patients with sick sinus syndrome is very common and is associated primarily with the initial presence of brady-tachy syndrome, with ventricular pacing, and with the occurrence of atrial fibrillation during follow up. M mode echocardiography is of no particular value in predicting thromboembolism in this population. The risk of thromboembolism is small in patients with atrial pacing in whom atrial fibrillation has never been observed.

The study was supported by grants from the Danish Heart Foundation and from Sygekassernes Helsefond. JCN has a grant from the Faculty of Health Sciences, University of Aarhus, Denmark.

1 Andersen HR, Nielsen JC, Thomsen PE, et al. Long-term follow up of patients from a randomised trial of atrial versus ventricular pacing for sick sinus syndrome. Lancet 1997;350:1210-16

2 Nielsen JC, Andersen HR, Thomsen PE, et al. Heart failure and echocardiographic changes during long term follow up of patients with sick sinus syndrome randomized to single-
chamber atrial or ventricular pacing. Circulation 1998;97: 987-95.

3 Andersen HR, Thuesen L, Bagger JP, et al. Prospective randomised trial of atrial versus ventricular pacing in sick-sinus syndrome. Lancet 1994;344:1523-8.

4 Rubenstein JJ, Schulman CL, Yurchak PM, et al. Clinical spectrum of the sick sinus syndrome. Circulation 1972;46: 5-13.

5 Sahn DJ, DeMaria A, Kisslo J, et al. Recommendations regarding quantitation in M-mode echocardiography:
results of a survey of echocardiographic measurements. results of a survey of echocard
Circulation 1978;58:1072-83.

6 Sgarbossa EB, Pinski SL, Maloney JD, et al. Chronic atrial fibrillation and stroke in paced patients with sick sinus syndrome. Relevance of clinical characteristics and pacing modalities. Circulation 1993;88:1045-53.

7 Stangl K, Seitz K, Wirtzfeld A, et al. Differences between atrial single chamber pacing (AAI) and ventricular single chamber pacing (VVI) with respect to prognosis and antiarrhythmic effect in patients with sick sinus syndrome. PACE 1990;13:2080-5.

8 Brandt J, Anderson H, Fahraeus T, et al. Natural history of sinus node disease treated with atrial pacing in 213 patients: implications for selection of stimulation mode. $\mathcal{F}$ Am Coll Cardiol 1992;20:633-9.

9 Rosenqvist $M$, Brandt J, Schuller H. Long-term pacing in sinus node disease: effects of stimulation mode on cardiovascular morbidity and mortality. Am Heart $f$ 1988;116:16-22.

10 Santini M, Alexidou G, Ansalone G, et al. Relation of prognosis in sick sinus syndrome to age, conduction defects and modes of permanent cardiac pacing. Am $\mathcal{F}$ Cardiol 1990;65: 729-35.
11 Sasaki Y, Shimotori M, Akahane K, et al. Long-term follow up of patients with sick sinus syndrome: a comparison of
clinical aspects among unpaced, ventricular inhibited paced, and physiologically paced groups. PACE 1988;11: 1575-83.

12 Fairfax AJ, Lambert CD, Leatham A. Systemic embolism in chronic sinoatrial disorder. N Engl f Med 1976;295:190-2.

13 Santini M, Ansalone G, Auriti A. Sick sinus syndrome: natural history before and after pacing. Eur $\mathcal{F}$ Card Physiol Electrophysiol 1993;3:220-31.

14 Sutton R, Kenny RA. The natural history of sick sinus syndrome. PACE 1986;9:1110-14.

15 Fisher M, Kase CS, Stelle B, et al. Ischemic stroke after cardiac pacemaker implantation in sick sinus syndrome. Stroke 1988;19:712-15.

16 Santini M, Ansalone G, Auriti A, et al. Stroke in sinus node disease. In: Rosenqvist M, ed. Cardiac pacing: new advances.

17 Atrial Fibrillation Investigators. Risk factors for stroke and efficacy of antithrombotic therapy in atrial fibrillation. efficacy of antithrombotic therapy in atrial fibrillation.
Analysis of pooled data from five randomized controlled Analysis of pooled data from five rando

18 Sethi KK, Bajaj JC, Mohan JC, et al. Comparison of atrial and VVI pacing modes in symptomatic sinus node dysfunction without associated tachyarrhythmias. Indian Heart F 1990;42:143-7.

19 Sasaki S, Takeuchi A, Ohzeki M, et al. Long-term follow up of paced patients with sick sinus syndrome. In: Steinbach K, Glokar D, Laszkovics A, et al, eds. Cardiac pacing. Proceedings of the VIIth world symposium on cardiac pacing. Darmstadt: Steinkopff Verlag, 1983:85-90.

20 The Stroke Prevention in Atrial Fibrillation Investigators. Predictors of thromboembolism in atrial fibrillation: I. Clinical features of patients at risk. Ann Intern Med 1992;116:1-5.

21 Cabin HS, Clubb KS, Hall C, et al. Risk for systemic embolization of atrial fibrillation without mitral stenosis. Am 7 Cardiol 1990;65:1112-16.

22 Benjamin EJ, D'Agostino RB, Belanger AJ, et al. Left atrial size and the risk of stroke and death. The Framingham heart study. Circulation 1995;92:835-41

23 Fazio S, Cittadini A, Sabatini D, et al. Platelet aggregability in patients with a VVI pacemaker. PACE 1993;16:254-6.

24 Lau CP, Tse H, Cheng G. Effects of atrioventricular asynchrony on platelet activation: implication of thromboembolism in paced patients. Heart 1997;78:358-63.

25 Simantirakis EN, Parthenakis FI, Chrysostomakis SI, et al. Left atrial appendage function during DDD and VVI pacing. Heart 1997;77:428-31.

26 Lee T-M, Su S-F, Chien K-L, et al. Left atrial spontaneous echo contrast in patients with permanent pacemakers. Heart 1997;78:262-7.

27 Fazio S, Santomauro M, Cittadini A, et al. Efficacy of ticlopidine in the prevention of thromboembolic events in pidine in the prevention of thromboembolic events

28 The Boston Area Anticoagulation Trial for Atrial Fibrillation Investigators. The effect of low-dose warfarin on the risk of stroke in patients with nonrheumatic atrial fibrillation. N Engl f Med 1990;323:1505-11.

29 Stroke Prevention in Atrial Fibrillation Investigators. Stroke prevention in atrial fibrillation study. Final results. Circulation 1991;84:527-39.

30 Roy D, Marchand E, Gagne P, et al. Usefulness of anticoagulant therapy in the prevention of embolic complications of atrial fibrillation. Am Heart f 1986;112:1039-43.

31 Orsinelli DA. Current recommendations for the anticoagulation of patients with atrial fibrillation. Prog Cardiovasc Dis 1996;39:1-20.

32 Munschauer FE, Priore RL, Hens M, et al. Thromboembolism prophylaxis in chronic atrial fibrillation. Practice patterns in community and tertiary-care hospitals. Stroke patterns in com

33 Lip GY, Tean KN, Dunn FG. Treatment of atrial fibrillation in a district general hospital. Br Heart $\mathcal{F} 1994 ; 71: 92-5$.

34 Antani MR, Beyth RJ, Covinsky KE, et al. Failure to prescribe warfarin to patients with nonrheumatic atrial fibrillation. F Gen Intern Med 1996;11:713-20.

35 Whittle J, Wickenheiser L, Venditti LN. Is warfarin underused in the treatment of elderly persons with atrial fibrillation? Arch Intern Med 1997;157:441-5.

36 Goldstein LB, Farmer A, Matchar DB. Primary care physician-reported secondary and tertiary stroke prevention practices. A comparison between the United States and the United Kingdom. Stroke 1997;28:746-51.

37 Wolf PA, Abbott RD, Kannel WB. Atrial fibrillation: a major contributor to stroke in the elderly. The Framingham contributor to stroke in the elderly. The
study. Arch Intern Med 1987;147:1561-4.

38 Petersen P, Boysen G, Godtfredsen J, et al. Placebocontrolled, randomised trial of warfarin and aspirin for prevention of thromboembolic complications in chronic atrial fibrillation. The Copenhagen AFASAK study. Lancet 1989;i:175-9. 